\title{
Compreensão das mães de uma estratégia de saúde da família do município de São João da Ponte/MG sobre o aleitamento materno
}

\author{
Mother understands of a family health strategy of the city of São Joao da Ponte/MG about \\ breastfeeding \\ Comprensión de las madres de una estrategia de salud de la familia del condado de São João da \\ Ponte/MG sobre la lactancia materna
}

Ana Luíza Quintino Spínola ${ }^{1}$; Eliane Campos Oliva Mont’Alvão² ${ }^{2}$ Patrick Leonardo Nogueira da Silva ${ }^{3}$ José Ronivon Fonseca ${ }^{4}$; Wilson Aguiar Filho ${ }^{5}$; Valessa Gizele Ramos de Oliveira ${ }^{6}$

\begin{abstract}
Resumo
O aleitamento materno é a estratégia isolada que mais previne mortes infantis, além de promover a saúde física, mental e psíquica da criança e da mulher que amamenta. Objetivou-se identificar a compreensão das mães de uma estratégia saúde da família do município de São João da Ponte/MG sobre aleitamento materno. Trata-se de um estudo descritivo, observacional, com abordagem quantitativa, realizado com 31 mães cadastradas em uma unidade básica de saúde. Utilizou-se uma entrevista semiestruturada na qual o tratamento dos dados se deu por meio de estatística não paramétrica. Predominaram-se mulheres entre 18-27 anos, ensino médio completo, um salário mínimo, sem vínculo empregatício, companheiro estável e dois filhos; 90,3\% relataram a prevenção de infecções como benefício da amamentação da criança; 51,6\% relataram a prevenção de doenças como benefício para as mesmas; $93,5 \%$ afirmam que a criança deve mamar sempre que desejar durante o dia; $67,7 \%$ conhecem os direitos garantidos por lei para nutrizes com vínculo empregatício; e $87,0 \%$ amamentaram exclusivamente até os seis meses. Conclui-se que as participantes possuem conhecimento satisfatório sobre os aspectos gerais da amamentação devendose a equipe de saúde expandir a promoção desta prática.
\end{abstract}

\footnotetext{
${ }^{1}$ Enfermeira pelas Faculdades Unidas do Norte de Minas (FUNORTE). Avenida Osmane Barbosa, n ${ }^{\circ} 11.111$, JK, Montes Claros, MG, Brasil. CEP: 39.404-549. E-mail: izaspinola@ hotmail.com

${ }^{2}$ Enfermeira pelas Faculdades Unidas do Norte de Minas (FUNORTE). Avenida Osmane Barbosa, $\mathrm{n}^{\circ}$ 11.111, JK, Montes Claros, MG, Brasil. CEP: 39.404-549. E-mail: elianemontalvao@ hotmail.com

${ }^{3}$ Mestrando em Saúde, Sociedade e Ambiente (Área de Concentração em Interdisciplinar e Linha de Pesquisa em Promoção da Saúde, Prevenção e Controle de Doenças) pela Universidade Federal dos Vales do Jequitinhonha e Mucuri (UFVJM). Professor da Escola Técnica de Saúde do Centro de Educação Profissional e Tecnológica. Rua Coronel Celestino, nº 65, Centro, Montes Claros, MG, Brasil. CEP: 39.400-014. E-mail: patrick_mocesp70@ hotmail.com

${ }^{4}$ Mestre em Cuidado Primário em Saúde (Área de Concentração em Saúde Coletiva e Linha de Pesquisa em Epidemiologia e Vigilância em Saúde) pela Universidade Estadual de Montes Claros (UNIMONTES). Professor do curso de graduação em Enfermagem das Faculdades Unidas do Norte de Minas (FUNORTE). Avenida Osmane Barbosa, n 11.111, JK, Montes Claros, MG, Brasil. CEP: 39.404-549. E-mail: pisecfunorte@ yahoo.com.br

${ }^{5}$ Mestre em Saúde Pública (Área de Concentração em Política, Planejamento, Gestão e Práticas em Saúde e Linha de Pesquisa em Saúde Coletiva) pela Escola Nacional de Saúde Pública Sérgio Arouca (ENSP). Servidor público da Fundação Oswaldo Cruz (FIOCRUZ). Rua Leopoldo Bulhões, n $1.480,7^{\circ}$ Andar, Sala 705, Manguinhos, Rio de Janeiro, RJ, Brasil. CEP: 21.041-210. E-mail: aguiar.wilson@ gmail.com

${ }^{6}$ Mestre em Ciências da Saúde (Área de Concentração em Promoção, Prevenção e Reabilitação em Saúde e Linha de Pesquisa em Estratégias Interdisciplinares em Promoção, Prevenção e Reabilitação em Saúde) pela Universidade Federal de São Paulo (UNIFESP). Professora do curso de graduação em Enfermagem das Faculdades Unidas do Norte de Minas (FUNORTE). Avenida Osmane Barbosa, n 11.111, JK, Montes Claros, MG, Brasil. CEP: 39.404-549. E-mail: valessagiz@yahoo.com.br
} 
ISSN 2179-6750

Descritores: Saúde Pública, Programa Saúde da Família, Saúde Materno-Infantil, Aleitamento Materno, Conhecimento.

\begin{abstract}
Breastfeeding is the single strategy that most prevents infant deaths, as well as promoting the physical, mental and psychological health of the child and the woman who breastfeeds. The objective of this study was to identify mothers' understanding of a family health strategy in the city of São João da Ponte/MG on breastfeeding. This is a descriptive study, observational, with a quantitative approach, performed with 31 mothers enrolled in a basic health unit. A semi-structured interview was used in which the data treatment was done through non-parametric statistics. Women aged between 18 and 27 years old were enrolled, with a high school, a minimum wage, no employment relationship, stable companion and two children; $90.3 \%$ reported the prevention of infections as a benefit of breastfeeding the child; $51.6 \%$ reported disease prevention as a benefit to them; $93.5 \%$ state that the child should nurse whenever he wishes during the day; $67.7 \%$ are aware of the rights guaranteed by law for nursing mothers with an employment relationship; and $87.0 \%$ exclusively breastfed until six months. It is concluded that the participants have satisfactory knowledge about the general aspects of breastfeeding, and the health team should expand the promotion of this practice.

Key-words: Public Health, Family Health Program, Maternal and Child Health, Breastfeeding, Knowledge.
\end{abstract}

\title{
Resumen
}

La lactancia materna es la estrategia aislada que más previene muertes infantiles, además de promover la salud física, mental y psíquica del niño y de la mujer que amamanta. Se objetivó identificar la comprensión de las madres de una estrategia salud de la familia del municipio de São João da Ponte/MG sobre lactancia materna. Se trata de un estudio descriptivo, observacional, con abordaje cuantitativo, realizado con 31 madres registradas en una unidad básica de salud. Se utilizó una entrevista semiestructurada en la cual el tratamiento de los datos se dio por medio de estadística no paramétrica. Se predijeron mujeres entre 18-27 años, enseñanza media completa, un salario mínimo, sin vínculo laboral, compañero estable y dos hijos; el 90,3\% relató la prevención de infecciones como beneficio de la lactancia materna; el 51,6\% relató la prevención de enfermedades como beneficio para las mismas; el 93,5\% afirma que el niño debe mamar siempre que desee durante el día; $67,7 \%$ conocen los derechos garantizados por ley para las mujeres con vínculo laboral; y el 87,0\% amamantó exclusivamente hasta los seis meses. Se concluye que las participantes tienen conocimiento satisfactorio sobre los aspectos generales de la lactancia, debiendo el equipo de salud expandir la promoción de esta práctica.

Palabras-claves: Salud Pública, Programa de Salud de la Familia, Salud Materna y del Niño, la lactancia materna, el conocimiento.

\section{Introdução}

Atualmente, é preconizado que o aleitamento materno (AM) seja exclusivo até os seis meses de vida do lactente e complementado até os dois anos ou mais. Considera-se aleitamento materno exclusivo (AME) o bebê que se alimenta apenas de leite humano, sem qualquer outro alimento, inclusive chás ou sucos ${ }^{1,2}$. A amamentação, quando praticada de forma exclusiva até os seis meses 
ISSN 2179-6750

e complementada com alimentos apropriados até os dois anos de idade ou mais, demonstra grande potencial transformador no crescimento e desenvolvimento $(\mathrm{CD})$ e previne doenças na infância e idade adulta e que, por essas e outras razões, é prioridade a criação de várias ações de promoção da amamentação ${ }^{3}$. O AM é a estratégia isolada que mais previne óbitos infantis, além de promover a saúde física, mental e psíquica da criança e da mulher que amamenta ${ }^{4}$. Esta é a primeira prática alimentar a ser estimulada para promoção da saúde, criando, assim, hábitos alimentares saudáveis e prevenindo muitas doenças ${ }^{5}$. Sendo assim, a recomendação nacional é a mesma e é a adotada no Estado de Minas Gerais (MG).

Poucas condições contraindicam a amamentação. São elas: mães com doenças graves, crônicas ou debilitantes; mães infectadas pelo Human Immunodeficiency Virus (HIV) ou vírus da imunodeficiência humana (VIH); mães que precisem tomar medicamentos imprescindíveis e por tempo prolongado na qual são nocivos aos bebês; e, ainda, bebês com doenças metabólicas raras, tal como a fenilcetonúria e a galactosemia ${ }^{6,7}$. É comprovado que a educação e o preparo das mulheres para a lactação durante o período pré-natal contribuem para o sucesso do AM, momento em que elas devem ser informadas sobre os benefícios da amamentação, das desvantagens do uso de leite não humano e quanto às técnicas de amamentação, para aumentar a sua habilidade e confiança ${ }^{8}$.

Estudo sugere que uma boa técnica de amamentação nos primeiros dias após o parto está associada ao aumento da duração do $\mathrm{AM}^{9}$. A falta de compreensão sobre o AM por parte das mães tem representado um papel importante na redução da duração dessa prática ${ }^{10}$. Logo, percebe-se que as Unidades Básicas de Saúde (UBS) têm papel fundamental no estímulo ao AM, especialmente através das Estratégias Saúde da Família (ESF). Algumas regiões menos favorecidas do norte e nordeste contaram inicialmente com o Programa de Agentes Comunitários de Saúde (PACS) instituído a partir de 1991 a fim de se obter um acompanhamento melhor das famílias de uma determinada área e reduzir a mortalidade materno-infantil.

As ESF foram instituídas em 1994 seguindo os mesmos padrões do PACS, atendendo as áreas mais pobres dos municípios onde havia maior dificuldade de acesso aos serviços de saúde e, devido à grande aceitação e bons resultados obtidos, houve investimentos significativos e elas passaram a ser adotadas em todo o território nacional ${ }^{11}$.

Sendo assim, este estudo objetivou identificar a compreensão das mães de uma ESF do município de São João da Ponte/MG sobre AM.

\section{Materiais e Métodos}

Trata-se de um estudo descritivo, observacional, com abordagem quantitativa. $\mathrm{O}$ mesmo foi 
ISSN 2179-6750

realizado na ESF Geraldo Gorutuba, localizada no Bairro Denise do município de São João da Ponte, MG, Brasil. Sua população estimada em 2013 era de 30.362 habitantes. Sua estrutura geográfica localiza-se na mesorregião do norte de MG e microrregião da cidade de Montes Claros na qual possui 11 ESF implantadas. Foi enviada uma carta de apresentação e um Termo de Consentimento Institucional (TCI) à Secretaria Municipal de Saúde (SMS) para autorização do estudo. A instituição foi devidamente orientada quanto às diretrizes da pesquisa e a mesma assinou o TCI de modo a autorizar a realização da pesquisa.

A ESF apresenta 906 famílias cadastradas, com oito microáreas, e abrange, no seguimento urbano, os Bairros Denise, Cruzeiro, Alvorada e Lagoinha, e na zona rural abrange as regiões de São Vicente, Barreiro Novo, Barreiro do Campo, Varginha, Ribeirão da Varginha, Ribeirão do Ouro e Tigre. A equipe é composta por médico, enfermeiro, cirurgião-dentista, técnico em saúde bucal (TSB), técnico em enfermagem, auxiliar em saúde bucal (ASB) e agentes comunitários de saúde (ACS). Além disso, recebem o apoio do Núcleo de Apoio à Saúde da Família (NASF) e de seus especialistas em nutrição, psicologia, fonoaudiologia, fisioterapia, farmácia e assistência social.

Os sujeitos da pesquisa foram compostos por 31 mães cadastradas na ESF, com filhos de até dois anos de vida no momento da coleta de dados. As mesmas apresentavam idade entre 18 e 37 anos. Essas mães foram identificadas por meio do Sistema de Informação de Nascidos Vivos (SINASC) na qual fizeram todo o seu acompanhamento pré-natal na ESF durante o período de 2009 a 2011. Foram adotados os seguintes critérios de inclusão: possuir filhos com idade de até dois anos; ser cadastrada na ESF. Quanto aos critérios de exclusão: não ser encontrada em sua residência após três tentativas; e mães com impossibilidade de responder por si mesma. Foi utilizada toda a amostra disponível.

Foi utilizado como instrumento de coleta de dados uma entrevista semiestruturada de elaboração própria contendo questões objetivas e subjetivas relativas à caracterização da amostra e à compreensão das mães sobre o AM. Após a coleta, foi realizada a tabulação com o Statistical Package for the Social Sciences (SPSS), versão 13.0, para posterior análise e discussão dos dados através de epidemiologia descritiva tendo como base o referencial teórico levantado. Os dados tabulados foram apresentados em tabelas, sendo a idade agrupada por conveniência conforme intervalo de 10 anos.

Os dados foram coletados no mês de novembro de 2011, no domicílio das mães, após leitura e assinatura do Termo de Consentimento Livre e Esclarecido (TCLE). O estudo obedeceu aos preceitos éticos estabelecidos pela Resolução no 466/2012, do Conselho Nacional de Saúde (CNS), na qual regulamenta pesquisas envolvendo seres humanos. O projeto de pesquisa foi apreciado e 
ISSN 2179-6750

aprovado pelo Comitê de Ética em Pesquisa das Faculdades Unidas do Norte de Minas (CEP FUNORTE), protocolo $n^{\circ}$ 01700/2011, CAAE: 0136.0.445.000-11/SISNEP.

\section{Resultados}

De acordo a Tabela 1, a maior parte das entrevistadas apresenta idade entre 18-27 anos (77,4\%); Ensino Médio completo (35,4\%); ganha apenas um salário mínimo (41,9\%); não apresentam vínculo empregatício (80,7\%); 96,7\% possuem companheiro estável; e 51,6\% possuem dois filhos.

Com relação ao tempo ideal de AME, $100 \%$ das entrevistadas relataram ser até os seis primeiros meses de vida da criança. Quanto ao aleitamento complementado, todas afirmaram que deve ser oferecido à criança após o período de AME indo até os dois anos de vida.

Tabela 1. Perfil socioeconômico e demográfico das mães. São João da Ponte (MG), 2011

\begin{tabular}{l|c|c}
\hline Variáveis & $\mathbf{n = 3 1}$ & $\mathbf{\%}$ \\
\hline Intervalo etário & & \\
\hline$[18,27]$ & 24 & 77,4 \\
\hline$[28,37]$ & 07 & 22,6 \\
\hline Escolaridade & & \\
\hline EF incompleto & 10 & 32,2 \\
\hline EF completo & 09 & 29,0 \\
\hline EM incompleto & 00 & 0,0 \\
\hline EM completo & 11 & 35,4 \\
\hline Menos de 01 ano de estudo & 01 & 3,4 \\
\hline Renda familiar & & \\
\hline Menor que 01 SM & 11 & 35,4 \\
\hline 01 SM & 13 & 41,9 \\
\hline Maior que 01 SM & 07 & 22,7 \\
\hline Vínculo empregatício & & \\
\hline Sim & 06 & 19,3 \\
\hline Não & 25 & 80,7 \\
\hline Situação conjugal & & \\
\hline Possui companheiro & 30 & 96,7 \\
\hline Não possui companheiro & 01 & 3,3 \\
\hline Quantidade de filhos & & \\
\hline 1 filho & 11 & 35,4 \\
\hline 2 filhos & 04 & 51,6 \\
\hline 3-5 filhos & &
\end{tabular}

Fonte: Pesquisa direta

Legenda:

EF = Ensino Fundamental; EM = Ensino Médio; SM = Salário Mínimo

Quanto à importância da amamentação para a criança, das 31 entrevistadas, 28 relataram a “prevenção de infecções"; 13 abordaram "ser um alimento saudável”; e 11 afirmaram "auxiliar no crescimento e desenvolvimento da criança", o que mostra que seus principais benefícios foram 
ISSN 2179-6750

relatados, embora não por todas as participantes (Tabela 2). Ainda de acordo a mesma tabela, a prevenção de doenças como importância da amamentação para a mãe foi afirmada por 16 das participantes, seguido do aumento do vínculo entre mãe e filho relatado por 12 mulheres e, por fim, da perda de peso relatado por 11 das mesmas. $\mathrm{O}$ único benefício que não foi relatado por nenhuma das participantes foi a economia financeira.

Quando questionadas sobre sua compreensão a respeito das situações que contraindicam a amamentação, a presença de infecções/doença foi questionada por 20 mulheres. Outros fatores alegados foram o uso de medicamentos $(n=3)$, mães portadoras de câncer de mama $(n=3)$, gravidez $(n=3)$, uso de prótese de silicone $(n=1)$, e cinco afirmaram desconhecer tais situações (Tabela 2). Salienta-se que nas questões oriundas da Tabela 2, as entrevistadas puderam escolher mais de uma opção como resposta.

Tabela 2. Importância e contraindicação da amamentação segundo a compreensão das mães. São João da Ponte (MG), 2011.

\begin{tabular}{l|c}
\hline Variáveis & $\mathbf{N}^{0}$ de vezes citados \\
\hline Importância da amamentação para a criança & \\
\hline Prevenção de infecções & 28 \\
\hline Ser um alimento saudável & 13 \\
\hline Auxilia no CD da criança & 11 \\
\hline Importância da amamentação para a mãe & 11 \\
\hline Perda de peso & 12 \\
\hline Aumento do vinculo mãe e filho & 16 \\
\hline Prevenção de doenças & 20 \\
\hline Contraindicação para a amamentação & 10 \\
\hline Infecções/Doenças & 05 \\
\hline Outros & \\
\hline Desconhece & \\
\hline
\end{tabular}

Fonte: Pesquisa direta

Em se tratando do tempo em que a criança deve mamar seguidamente em uma mama e da periodicidade da troca da mama, todas as participantes (100\%) referiram que a criança deve mamar na mesma mama até soltar o peito e que se deve trocar de mama na próxima mamada, respectivamente. Quando perguntadas quantas vezes a criança deve mamar, 93,5\% responderam que deve mamar sempre que desejar durante o dia e 77,4\% relataram que deve mamar sempre que desejar durante a noite, o que demonstra que as mães possuem uma compreensão adequada em relação às recomendações vigentes do Ministério da Saúde (MS) sobre estas questões abordadas (Tabela 3).

Dentre as entrevistadas, $21(67,7 \%)$ relataram conhecer os direitos garantidos por lei para mães e nutrizes com vínculo empregatício e 10 (32,3\%) relataram desconhecer sobre estes direitos. 
As mães foram abordadas com relação ao recebimento de orientações sobre amamentação na UBS. Todas (100\%) argumentaram que foram instruídas pela ESF em que são cadastradas sobre amamentação. Quando questionadas sobre ter realizado o pré-natal para o último filho ou não ter realizado, $100 \%$ das mulheres entrevistadas disseram ter realizado pelo menos seis consultas de prénatal na ESF Geraldo Gorutuba, o que juntamente com as orientações feitas pela UBS provavelmente contribuiu para que as mães apresentassem uma compreensão adequada sobre o AM.

Foi investigada sobre a prática de AME tal como mostra a Tabela 3. A maior parte das entrevistadas (87\%) afirmou ter amamentado ou estarem amamentando seu último filho menor de seis meses de forma exclusiva. Vinte e três crianças já possuem mais de seis meses. Destas, 19 estão em aleitamento complementado e quatro já foram desmamadas.

Tabela 3. Compreensão das técnicas, da periodicidade da amamentação e do AME nos primeiros seis meses. São João da Ponte (MG), 2011

\begin{tabular}{l|c|c}
\hline Variáveis & $\mathbf{n = 3 1}$ & $\mathbf{\%}$ \\
\hline Tempo em que a criança deve mamar seguidamente em uma mama & & \\
\hline Até “soltar” o peito & 31 & 100 \\
\hline Outras respostas & - & - \\
\hline Quando se deve trocar de mama & & \\
\hline Na próxima mamada & 31 & 100 \\
\hline Na mesma mamada & - & - \\
\hline Quantas vezes a criança deve mamar durante o dia & & \\
\hline De 4-6 vezes & 02 & 6,5 \\
\hline Sempre que desejar & 29 & 93,5 \\
\hline Quantas vezes a criança deve mamar durante a noite & & \\
\hline Até três vezes & 07 & 22,6 \\
\hline Sempre que desejar & 24 & 77,4 \\
\hline AME dos filhos durante os seis primeiros meses & & \\
\hline Sim & 27 & 87 \\
\hline Não & 04 & 13 \\
\hline Fonte: Pesquisa direta & &
\end{tabular}

\section{Discussão}

Os aspectos socioeconômicos e demográficos contribuem para uma melhor compreensão das informações quanto ao $\mathrm{AM}^{12}$. A experiência materna prévia na qual a maioria das entrevistadas tinha dois filhos ou mais; o grau de escolaridade materna na qual quanto melhor a escolaridade maior a compreensão; a influência da família, principalmente das avós e da mídia; dentre outros correlacionados neste estudo ajudam no aumento da qualidade do AM.

De acordo com o preconizado pelo MS, entende-se que a alimentação saudável começa com o AM, exclusivamente oferecida ao lactente até os seis meses de vida e complementando até os dois anos de idade ou mais ${ }^{13}$. Já está devidamente comprovada, por estudos científicos, a superioridade do leite materno sobre os leites de outras espécies, pois ele possui fatores capazes de proteger contra 
ISSN 2179-6750

infecções e diarreia ${ }^{14}$, principalmente em crianças de baixo poder aquisitivo e diminui a gravidade dos episódios de infecções respiratórias. O AME diminui ainda o risco de Hipertensão Arterial Sistêmica (HAS), Diabetes Mellitus (DM) e hipercolesterolemia, previnem o aparecimento de alergias, reduz a chance de obesidade e também confere uma melhor nutrição, sendo uma importante fonte de nutrientes no segundo ano de vida, especialmente de proteínas, gorduras e vitaminas, e é capaz de suprir sozinhas as necessidades nutricionais da criança nos primeiros seis meses ${ }^{2}$. Sendo assim, os dados deste estudo convergem com os achados na literatura científica na qual as mães compreendem de forma adequada sobre os benefícios para a criança quanto para elas mesmas. O dado mais enfatizado pelas entrevistadas foi a proteção contra doenças ou infecções.

Outras vantagens do AME até os seis meses de vida incluem o efeito positivo na memória, contribuindo para o desenvolvimento cognitivo, melhor desenvolvimento da cavidade bucal devido ao exercício que a criança faz ao sugar o leite da mama, auxilia a evitar uma nova gravidez, pois as mulheres que ovulam antes do sexto mês após o parto, em geral, amamentam menos vezes por dia que as demais ${ }^{4}$. Mesmo conhecendo-se a grande importância do AM, em alguns casos pode haver a necessidade de inibir ou suprimir a produção de leite, em especial quando há presença de certas doenças na mulher ${ }^{6}$. Existem contraindicações temporárias e definitivas. Existem certas situações em que as mães não devem amamentar temporariamente os seus bebês até que elas se resolvam, sendo alguns exemplos: mães com algumas doenças infecciosas tal como a varicela e a herpes; com lesões mamárias; uso de medicação imprescindível. As contraindicações absolutas à amamentação natural são mães que apresentam doenças graves, crônicas ou debilitantes ${ }^{7}$. Neste estudo, a contraindicação mais abordada foi por doença ou infecção, porém as mesmas não especificam o tipo da patologia e outras desconhecem as contraindicações de forma a tornar-se um fator de risco para a criança.

Os procedimentos estéticos nas mamas são recentes do ponto de vista da ciência e há poucos estudos avaliando o impacto da mamoplastia de aumento na lactogênese e amamentação de forma geral. Existem certas cirurgias que podem afetar a mama e, conseqüentemente, a produção do leite materno, mas a colocação do silicone não costuma ser uma delas. Neste caso, pode haver dificuldade de amamentação se as próteses aumentarem muito a pressão intramamária ${ }^{15}$. O horário não é o mais importante. O bebê deve ser alimentado sempre que tiver fome, tanto de dia quanto de noite, pelo tempo que quiser, o que é chamado de regime livre. Durante a noite, a prolactina tem um aumento na sua produção, portanto, amamentar durante a noite é especialmente importante para a produção de leite ${ }^{7}$. Com isso, as mães apresentam uma compreensão adequada conforme o que a literatura preconiza, bem como o MS. 
ISSN 2179-6750

O lactente não deve ser mudado de seio durante uma mesma mamada, mas somente na próxima, pois o leite tem constituições diferentes no início e no fim da mamada. O leite final é mais rico em gorduras e vitamina A, o que evita a desnutrição e doenças oftálmicas, respectivamente. Já o leite inicial é rico em água e sais minerais de forma a prevenir a desidratação, dentre outros problemas ${ }^{16}$.

Os direitos previstos pela Constituição Federal (CF) de 1988 e a Consolidação das Leis do Trabalho (CLT) estabelecem ${ }^{7,1}$ : licença após o parto de 120 dias (Art. $7^{\circ}$, inciso XVII da CF e Art. 395 da CLT); licença paternidade de cinco dias (Art. $7^{\circ}$, inciso XIX da CF), a partir do dia do nascimento do filho; direito a uma hora a menos em sua jornada de oito horas de trabalho para a amamentação ao retornar ao trabalho, até a criança completar seis meses, sob a forma de dois intervalos de meia hora em cada turno ou, opcionalmente, uma hora a menos na entrada ou saída do expediente (Art. 396 da CLT); garantia do emprego à gestante, desde a confirmação da gravidez até o quinto mês após o parto, incluindo aviso prévio (Art. 10, inciso II do Ato das Disposições Constitucionais Transitórias - CF); garantia de creche para os filhos de até seis anos das trabalhadoras por parte das empresas com mais de 30 funcionárias (Art. 389, 397 e 400 da CLT). No estudo, quase todas as entrevistadas tem ciência de seus direitos.

As ações sistematizadas de incentivo ao AM devem estar incorporadas às atividades de rotina das UBS e maternidades, devendo abranger o pré-natal, o parto e o primeiro ano de vida ${ }^{1}$. A importância da UBS na abordagem sobre o AM pode ser realizada por meio da ESF nas ações de pré-natal, acompanhamento do $\mathrm{CD}$, teste do pezinho, imunização e no planejamento familiar. Acrescentam, ainda, que a ESF deve proporcionar às famílias, principalmente às mães, orientações sobre o AM durante as visitas domiciliares, contribuindo para que os processos de incentivo ao AM possam ser oferecidos de maneira efetiva e a população possa estar devidamente assistida pelos serviços de saúde ${ }^{17}$. O pré-natal é um momento importante em que o profissional de saúde informa a gestante dos benefícios da amamentação, das desvantagens do uso de leite não humano e quanto às técnicas da amamentação, para aumentar a sua habilidade e confiança e garantir sucesso no processo de amamentação ${ }^{8}$.

\section{Conclusão}

A partir dos dados obtidos, pode-se perceber que a maior parte das mulheres são mães jovens, com idade ativa entre 18-27 anos, boa instrução escolar na qual a maior parte apresenta EM completo, não tem vínculo empregatício por estarem cuidando de seus filhos, apresentam companheiro estável e tem uma média de dois filhos. Com referência à compreensão sobre a 
ISSN 2179-6750

amamentação, as mães são bem informadas quanto o tempo ideal de AME, bem como o tempo em que a criança deve mamar seguidamente em uma mama, quando se deve trocar de mama, quantas vezes a criança deve mamar durante o dia ou à noite, e também sobre a importância desta prática para a criança e para a mãe. Dentre as mães entrevistadas, as mesmas mais desconheciam os direitos garantidos por lei para nutrizes e mães com vínculo empregatício e as situações que contraindicam a prática do AM.

Portanto, conclui-se que a compreensão das participantes sobre os aspectos gerais da amamentação está adequada em relação às recomendações do MS, fato que pode ter relação direta com as ações desenvolvidas pela ESF, sendo que todas elas relataram terem feito pré-natais e recebidas orientações podendo ter influenciado ainda mais na prática do AM, pois a maior parte das entrevistadas amamenta ou amamentaram seus filhos de forma exclusiva até os seis meses.

\section{Referências}

1. Minas Gerais. Secretaria de Estado de Saúde. Atenção à saúde da criança. Belo Horizonte (MG), 2004.

2. Brasil. Ministério da Saúde. Secretaria de Atenção à Saúde. Departamento de Atenção Básica. Saúde da criança: nutrição infantil - aleitamento materno e alimentação complementar. Brasília (DF), 2009.

3. Brasil. Ministério da Saúde. Secretaria de Atenção à Saúde. Departamento de Ações Programáticas e Estratégicas. Agenda de compromissos para a saúde integral da criança e redução da mortalidade infantil. Brasília (DF), 2004.

4. Brasil. Ministério da Saúde. Secretaria de Atenção à Saúde. Departamento de Ações Programáticas e Estratégicas. II Pesquisa de prevalência de aleitamento materno nas capitais brasileiras e Distrito Federal. Brasília (DF), 2009.

5. Brasil. Ministério da Saúde. Secretaria de Atenção à Saúde. Guia alimentar para a população brasileira: promovendo a alimentação saudável. Brasília (DF), 2008.

6. Carvalho JKM, Carvalho CG, Magalhães SR. A importância da assistência de enfermagem no aleitamento materno. e-Scientia. 2011;4(2):11-20.

7. Fundo das Nações Unidas para a Infância. Comitê Português para a Unicef. Comissão Nacional de Iniciativa dos Hospitais Amigos dos Bebês. Manual de aleitamento materno. Brasília (DF): Unicef, 2008. 
ISSN 2179-6750

8. Demitto MO, Silva TC, Páschoa ARZ, Mathias TAF, Bercini LO. Orientações sobre amamentação na assistência pré-natal: uma revisão integrativa. Rev RENE. 2010;11(esp):223-9.

9. Weigert EML, Giugliani ERJ, França MCT, Oliveira LD, Bonilha A, Santo LCE et al. The influence of breastfeeding technique on the frequencies of exclusive breastfeeding and nipple trauma in the first month of lactation. J Pediatr (Rio J). 2005;81(4):310-6.

10. Azeredo CM, Maia TM, Rosa TCA, Silva FF, Cecon PR, Cotta RMM. Percepção de mães e profissionais de saúde sobre o aleitamento materno: encontros e desencontros. Rev Paul Pediatr. 2008;26(4):336-44.

11. Silva FV, Menezes MDGAS. Formação profissional e humanização dos serviços de saúde. Rev Formação. 2002;5:59-74.

12. Esteves TMB, Daumas RP, Oliveira MIC, Andrade CAF, Leite IC. Factors associated to breastfeeding in the first hour of life: systematic review. Rev Saúde Pública. 2014;48(4):697-708.

13. Barreto SM, Pinheiro ARO, Sichieri R, Monteiro CA, Batista Filho M, Schimidt MI et al. Análise da estratégia global para alimentação, atividade física e saúde da Organização Mundial da Saúde. Epidemiol Serv Saúde. 2005;14(1):41-68.

14. Santos FS, Santos FCS, Santos LH, Leite AM, Mello DF. Breastfeeding and protection against diarrhea: an integrative review of literature. Einstein. 2015;13(3):435-40.

15. Dornaus MFPS. A experiência de amamentação de um grupo de mulheres com mamoplastia redutora e de aumento. São Paulo (SP). 104 fl. Dissertação [Mestrado em Enfermagem] Escola de Enfermagem, Universidade de São Paulo, 2005.

16. Ribeiro KDS, Dimenstein R. Níveis de retinol no leite materno ao início e final da mamada. Rev Panam Salud Publica. 2004;16(1):19-22.

17. Souza TO, Bispo TC. Aleitamento materno exclusivo e o Programa Saúde de Família da Chapada, município de Aporá (BA). Rev Baiana Saúde Pública. 2007;31(1):38-51. 